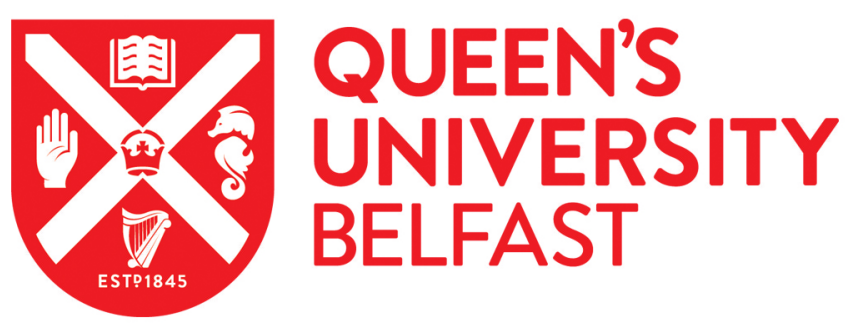

\title{
Attention during social interaction in children with autism: Comparison to specific language impairment, typical development, and links to cognition
}

Hanley, M., Riby, D. M., McCormack, T., Carty, C., Coyle, L., Crozier, N., Robinson, J., \& McPhillips, M. (2014). Attention during social interaction in children with autism: Comparison to specific language impairment, typical development, and links to cognition. Research in Autism Spectrum Disorders, 8(7), 908-924.

https://doi.org/10.1016/j.rasd.2014.03.020

\section{Published in:}

Research in Autism Spectrum Disorders

\section{Document Version:}

Peer reviewed version

Queen's University Belfast - Research Portal:

Link to publication record in Queen's University Belfast Research Portal

\section{Publisher rights}

( 2014 Elsevier Ltd. This manuscript version is made available under the CC-BY-NC-ND 4.0 license http://creativecommons.org/licenses/bync-nd/4.0/ which permits distribution and reproduction for non-commercial purposes, provided the author and source are cited.

\section{General rights}

Copyright for the publications made accessible via the Queen's University Belfast Research Portal is retained by the author(s) and / or other copyright owners and it is a condition of accessing these publications that users recognise and abide by the legal requirements associated with these rights.

Take down policy

The Research Portal is Queen's institutional repository that provides access to Queen's research output. Every effort has been made to ensure that content in the Research Portal does not infringe any person's rights, or applicable UK laws. If you discover content in the

Research Portal that you believe breaches copyright or violates any law, please contact openaccess@qub.ac.uk. 
Attention during social interaction in children with autism: Comparison to Specific Language Impairment, typical development, and links to social cognition

Mary Hanley ${ }^{1}$, Deborah M Riby ${ }^{2}$, Teresa McCormack ${ }^{1}$, Clare Carty ${ }^{1}$, Lisa Coyle ${ }^{1}$, Naomi Crozier $^{1}$, Johanna Robinson ${ }^{1}$, Martin McPhillips ${ }^{1}$.

\footnotetext{
${ }^{1}$ School of Psychology, Queen’s University Belfast, UK

${ }^{2}$ School of Psychology, Newcastle University, UK
}

\begin{abstract}
Acknowledgements
We are grateful for the kind cooperation of the participants involved in this study, their parents and schools. We acknowledge the support of Belvoir Park Primary School, Harberton Primary School, Thornfield Primary School, and St. Kieran’s Primary School. We also acknowledge the technical guidance provided by Dr Martin Sawey, School of Psychology, Queen’s University Belfast.
\end{abstract}

Correspondence to: Dr Mary Hanley, School of Psychology, Queen’s University, Belfast, BT7 1NN, UK. Telephone: 0289097 4886. Email: m.hanley@qub.ac.uk 


\section{Abstract}

Eye-tracking studies have shown how people with autism spend significantly less time looking at socially relevant information on-screen compared to those developing typically. This has been suggested to impact on the development of socio-cognitive skills in autism. We present novel evidence of how attention atypicalities in children with autism extend to reallife interaction, in comparison to typically developing (TD) children and children with Specific Language Impairment (SLI). We explored the allocation of attention during social interaction with an interlocutor, and how aspects of attention (awareness checking) related to traditional measures of social cognition (false belief attribution). We found divergent attention allocation patterns across the groups in relation to social cognition ability. Even though children with autism and SLI performed similarly on the socio-cognitive tasks, there were syndrome-specific atypicalities of their attention patterns. Children with SLI were most similar to TD children in terms of prioritizing attention to socially pertinent information (eyes, face, awareness checking). Children with autism showed reduced attention to the eyes and face, and slower awareness checking. This study provides unique and timely insight into real-world social gaze (a)typicality in autism, SLI and typical development, its relationship to socio-cognitive ability, and raises important issues for intervention.

Keywords:

Autism, Specific Language Impairment, eye-tracking, social interaction, implicit mentalising

Real-life social attention in children with Autism: Comparison to Specific Language Impairment, typical development, and links to social cognition

\section{Introduction}


Autism Spectrum Disorder (ASD) is a neurodevelopmental disorder characterised by impairments in social and communicative functioning, as well as the presence of repetitive / stereotyped patterns of behaviour (APA, 1994). Difficulties with reciprocal social interactions are among the greatest challenges that individuals with ASD face in their everyday activities, and this type of difficulty can lead to a range of negative outcomes, such as social isolation, anxiety and depression (Bellini, Peters, Bennet \& Hopf, 2007; Howlin, Goode, Hutton \& Rutter, 2004). In this study we focus on the nature of gaze to socially relevant information for individuals functioning on the autism spectrum. We specifically explore the importance of the nature of experimental stimuli, the importance of comparison groups, and the relationship between gaze behaviour and other socio-cognitive capabilities.

\subsection{Gaze behaviours and Autism}

Atypical social gaze is among the earliest clinical markers for ASD (Volkmar, Carter, Grossman \& Klin, 1997), which is important as typical social gaze is key to many early socio-developmental milestones, such as joint attention (von Hofsten \& Gredebäck, 2009). Attention to the eyes of others is significant for social development and social adaption in early childhood, but it is also important in later life, as much of our socialization is typically facilitated by information portrayed by the eyes (Klin, 2008; Jones, Carr \& Klin, 2008). It has been suggested that atypical attention to social information early in development could have a cascading effect on subsequent social development, derailing typical social learning, ultimately contributing to the development of atypical social behaviours that we associate with functioning on the autism spectrum (Dawson et al., 2004; Klin, 2008; Klin \& Jones, 2008; Pruett et al., 2011; Senju \& Johnson, 2009). Therefore, exploration of social gaze in 
autism has become an important research priority, with potential theoretical and clinical implications.

Research on social gaze (looking to faces) has provided an important gateway to understanding socio-cognitive difficulties in autism. For example, Dawson, Meltzoff, Osterling, Rinaldi and Brown (1998) found that children with autism failed to orient to social stimuli (e.g. name calling), but not non-social stimuli (e.g. jack-in-the-box), highlighting the fact that social information does not get attentional priority for children with autism. Mundy, Sigman, Ungerer and Sherman (1986) showed how deficits in joint attention observed during play-based interactions best discriminated children with autism from children with developmental delay, highlighting that joint attention is a key area of difficulty for children with autism.

Research on looking to faces has allowed better definition of the social deficits in autism, and advances with eye tracking technology have been central to furthering this literature. Whereas early studies of gaze behaviour in autism relied on less precise methods, such as behavioural coding from video, eye-tracking provides an accurate method of capturing spontaneous (or task directed) attention. It gives detailed moment to moment recordings of where and what an individual is attending to, and thus what information they have available to them to process. By capturing spontaneous attention allocation in experiments depicting social information (faces, people, social interaction), eye tracking studies have helped to explain the everyday social interaction difficulties faced by individuals with autism in a way that was not previously possible (Boraston \& Blakemore, 2007).

A range of eye tracking studies have shown how people with autism attend atypically to social stimuli (still images of faces or social scenes; clips of dynamic social interaction), mainly in terms of reduced attention to the eyes and faces of others (Corden et al., 2008; 
Pelphrey et al., 2002; Riby \& Hancock, 2009a; Riby \& Hancock, 2009b; Frazier Norbury et al., 2009; Klin, Jones, Schultz, Volkmar \& Cohen, 2002b; Nakano et al., 2010). When attention is allocated to a face, it seems that it is directed to the mouth region for an atypically increased time compared to individuals who do not have autism and this may be an indicator of reliance upon speech and language to understand social interactions (Jones et al., 2008; Klin et al., 2002b). A strong pattern to have also emerged is that of atypically increased attention to bodies, objects, and background regions of stimuli (Klin et al., 2002b; Rice, Moriuchi, Jones \& Klin, 2012). Therefore within a stimulus image individuals who are functioning on the autism spectrum do not distribute their attention to the same information as typically developing individuals. This may be especially the case when there is competition between social and non-social information (e.g. Speer et al., 2007). Furthermore, even when overall amount of attention allocation to regions of interest is typical, other aspects of social attention are atypical in autism, such as the timing of attention to social information and following another's gaze cues in social scenes (Fletcher-Watson et al., 2009; Freeth et al., 2010). Such findings shed some light on how the social world looks to a person with autism, and can help to explain some of the social difficulties people with autism face (e.g. understanding facial expressions, inappropriate social responses, greater interest in objects rather than people).

These attention patterns highlighted in research outlined above come from studies involving samples of people with autism ranging in age from 2 years (Jones et al., 2008); to school aged children (Riby \& Hancock, 2009a, 2009b; Rice et al., 2012); to adults with autism (Hanley, McPhillips, Mulhern \& Riby, 2012; Klin et al., 2002b); and from participants ranging in levels of cognitive functioning (Rice et al., 2012). Although very informative, important questions remain which could further the clinical utility of such research. The first is on the nature of atypicalities of social attention in autism, as although the patterns reported 
above are representative of the literature, there have been important inconsistencies, particularly in relation to attention the mouth in autism (Bar-Haim, Shulman, Lamy, \& Reuveni, 2006, and Rutherford \& Towns, 2008). Inconsistencies between reports of typical and atypical social attention are discussed in more detail in the next section. Furthermore, the way that atypical social attention in real-life contributes to the difficulties people with autism face (as opposed to attention while viewing social information on a screen) is as yet poorly understood. This is important given that the issue of ecological validity has been at the core of the debate on the nature of atypical social attention in autism.

A further key issue relates to the specificity of the atypical social attention profile associated with autism (particularly to eyes and mouth), as the majority of work in the area has involved typical comparison groups. Involving more appropriate comparison groups (who share some of the same difficulties as children with autism, e.g. communication difficulties) could help to elucidate the roots of atypical attention profiles and potential compensatory strategies. Finally, a critical next step for work in this area is to go beyond capturing atypical attention in autism, to exploring how this relates to social understanding and socio-cognitive capacities; we will return to this issue later in this introduction.

\subsection{Ecological validity - what is social about social interaction?}

Research on social attention in autism has involved the use of a range of social stimuli. Essentially stimuli have differed in the way faces appear or are presented within them, e.g. isolated faces; faces in social scenes; moving faces in clips of social interaction. Although reduced attention to the eyes is the most commonly reported pattern associated with autism, typical amounts of time viewing of the eyes has also been found (Fletcher-Watson, Leekam, Benson, Frank \& Findlay, 2009; Freeth, Chapman, Ropar \& Mitchell, 2010; van der Geest, Kemner, Verbaten, \& van Engeland, 2002). Emerging evidence indicates that stimuli 
characteristics can have an influence on attention patterns. Using a range of modern colour still images, Hanley et al. (2012) have shown how attention allocation to the eyes varies for the same group of high functioning individuals with Asperger Syndrome (AS) compared to typically developing (TD) participants, depending on the context within which faces are presented. Typical viewing of the eyes was reported when these participants with AS viewed faces presented in isolation (image cropped at shoulders against blank background), and reduced attention to the eyes was found when the same participants with AS viewed the same faces but in the context of a social scene (two individuals, bodies and background visible; Hanley et al., 2012). Speer et al. (2007) have also reported similar effects of stimuli characteristics on viewing patterns in autism. The evidence suggests that closer replication of realistic social information is critical in studies of social attention.

The very things that are social about social interaction have largely eluded eye tracking studies to date. Critical to social interaction is the interlocutor, engaging you in mutual gaze, taking their turn to speak to you, as well as to listen to you, all the while modulating their gaze and facial expressions accordingly (Kleinke, 1986; Riby, DohertySneddon \& Whittle, 2012). You, as the conversational partner must take your cue to respond to a question when asked, and show interest when listening by attending to the face of the interlocutor (Doherty-Sneddon, Riby \& Whittle, 2012). Paying attention to other's faces and eyes is critical for understanding during social interaction (as opposed to being distracted by objects), and picking up on social cues at the right time is important (Doherty-Sneddon et al., 2012; Riby et al., 2012). To understand the role of social attention atypicalities in autism, and to maximise potential clinical applications, it is imperative that we study real-life scenarios. The current study aims to address some of these key issues by measuring social attention in children with autism during a real-life interaction using eye tracking techniques. 
To our knowledge, only one other published study has attempted this. Nadig, Lee, Singh, Bosshart and Ozonoff (2010) explored verbal exchange and eye gaze behaviour in children with autism during a conversation with an adult. They explored the quality of verbal exchange across two topics of conversation (max of 3 minutes for each topic), a generic topic and a topic of circumscribed interest (CI) to the children, and they measured eye gaze behaviour from a subset of their participants during the conversation. There were several aspects of verbal exchange that were atypical for the children with autism, including maintaining topic of conversation, and making a greater proportion of atypical utterances which interfered with the flow of conversation. Quality of verbal exchange was found to be less reciprocal for the children with autism during the CI topic. However, both their participants with autism and the typical comparison children increased their gaze time to the adult's face during conversation on topics of CI, and importantly, they found no group differences in the time spent attending to specified regions of interest (whole face, body, nonpartner areas). This indicates that children with autism show typical social gaze during a real social scenario and seems to add to literature suggesting typical social gaze in individuals with autism even within a real-life interaction. However, the authors did not provide information on attention to face regions (eyes, nose, mouth etc.) which is very important given that we know attention to eyes and mouth has been found to be most atypical in autism (Klin et al., 2002b). Therefore, we aim to explore where children look during a real social scenario, especially in terms of face regions.

\subsection{Cross syndrome comparison - Specific Language Impairment}


The most commonly used comparison groups in research studies on autism are groups of typically developing individuals matched for age and cognitive ability. While this method of matching can indicate the typicality of attention patterns, a pertinent question relates to the 'uniqueness' of atypical attention patterns in autism by comparison to other developmental conditions (Burack, Iarocci, Flangan \& Bowler, 2004). This allows us to tap into the question of syndrome-specificity. The use of appropriate cross-syndrome comparisons can help to elucidate the relationships between underlying processes (in this case social attention) and autism symptomatology (Bishop \& Norbury, 2005).

A comparison that is rarely made in eye tracking research is between autism and specific language impairment (SLI). According to the diagnostic criteria outlined in DSM-IVTR (American Psychiatric Association (APA), 2000) ${ }^{1}$, SLI is characterised by receptive and expressive language difficulties which interfere with academic or occupational achievement or with social communication. However, the criteria for a pervasive developmental disorder must not be met (APA, 2000). Given that both groups share similar communication difficulties but differ markedly in terms of social functioning, this comparison offers an interesting way of exploring how different profiles of socio-communicative impairment impact on attention. For example, in relation to weak central coherence accounts of autism, Brock, Norbury, Einav and Nation (2008) explored the processing of words in context by individuals with autism by comparing their attention patterns to individuals matched for language ability (including participants with language impairments). By using this comparison, it was possible to show that reduced sensitivity to sentence context was not specific to autism, but mediated by language ability more generally (Brock et al., 2008).

\footnotetext{
${ }^{1}$ The DSM-IV-TR is referred to here as the participants with Autism and with SLI in the current research were diagnosed according to the DSM-IV-TR criteria.
} 
Thinking back to the previously mentioned atypical distribution of facial attention to the mouth by individuals with autism, a 'mouth bias' has been suggested to represent a strategy to compensate for social impairment because of previously reported associations with better social competence (Klin et al., 2002b). In other words, people with autism direct their attention to the mouth as a means of relying on speech and language to make sense of the social world. However, communication impairments also form part of the triad, and as such, could drive increased mouth attention. We know from the literature on typical development that visual cues from the face have an influence on speech perception. For example, focusing on lip movements can enhance the perception of speech when the quality of the speech is degraded (Varghese, Ozmeral, Best \& Shinn-Cunningham, 2012) and when talking on the telephone we need to modify our communication strategy (Doherty-Sneddon \& Kent, 1996). Therefore, it may be difficulties in communication (processing of speech and language) that drive a preferential attention bias to the mouth region in autism.

Indeed, research exploring different profiles of socio-communicative impairment within ASD and social attention highlight the link between communication skills and mouthlooking. Frazier Norbury et al. (2009) compared the social attention patterns of two groups of teenagers with autism - one group with autism and language impairment (ALI), and one group with autism but no language impairment (ALN) - and TD individuals. They suggested that if a mouth bias was a compensatory strategy to accrue social information from language, it would be expected that an ALN group might exhibit increased fixation to the mouth, while an ALI group would not, as increased fixation to the mouth for these individuals would not afford them additional information. Participants in this study viewed dynamic clips of a social interaction while their eye movements were recorded, and although an attentional bias for the mouth was not found for any of the groups, more looking at the mouth was associated with better communicative competence for participants with autism. Using eye-tracking 
techniques and presenting different kinds of social stimuli (static emotional faces, dynamic emotional faces, dynamic clips of an actor), Falck-Ytter , Fernell, Gillberg, and von Hofsten (2010) showed how social attention patterns distinguished different subgroups of children with autism according to their profile of socio-communicative impairment. Children with autism with better non-verbal communication than social interaction skills honed in on mouths and non-verbal communicative information (from an actor's body) than on eyes. The opposite pattern was found (honing in on eyes) for children with autism who had better social interaction skills than non-verbal communication skills. Therefore, the presence of a mouth bias, its specificity to autism and its possible underlying cause remains an issue for consideration. An important way of exploring this further is to compare the attention patterns of children with autism to children with SLI using eye tracking techniques.

Hosozawa, Tanaka, Shimizu, Nakano and Kitazawa (2012) and Nakano et al. (2010) have made this comparison between autism and SLI by comparing gaze data to the same dynamic social stimuli across two studies. The first study (Nakano et al., 2010) involved children and adults with autism and typical controls, and the second (Hosozawa et al., 2012). involved children with SLI and typical controls. When they compared gaze data from both studies, they found gaze behaviour between SLI and TD children to be very similar (largely directed towards faces), contrasting with the gaze behaviour of individuals with autism (reduced attention to faces). However, although both the TD and SLI groups spent very similar percentages of time looking at faces (TD: 70\%; SLI: 69\%), the distribution of attention between the eyes and mouth was quite different between the groups. Whereas the TD children spent 29\% attending the eye regions (for socio-emotional information) and 21\% attending the mouth regions (for audio-visual information), the SLI children spent a greater percentage of time viewing the mouth regions than the eyes (27\% versus $23 \%$ respectively). The authors suggested that this may represent a compensatory strategy in SLI making up for 
weak audio-visual processing, which may place children with SLI at risk for social difficulties. This indicates that a mouth bias could in fact be due to communication impairments, however if that account were feasible it is surprising that Hosozawa et al. (2012) such a bias was not present for young children with autism in Hosozawa et al (2012).

It is worth considering once more the demands of passively viewing interaction on a screen as opposed to participating in a real social interaction, which are very different. If a mouth bias exists and is related to social/communicative functioning, it may be more likely to be observed in real social interaction where it is incumbent upon the participant to process speech. Therefore, we aimed to explore gaze behaviour to an interlocutor's face (especially eyes and mouth) during a real-life social scenario. We aimed to explore this by comparing the attention patterns of children with autism to two comparison groups: a group of children with SLI matched for verbal ability; and a further group of TD children matched for non-verbal ability.

\subsection{Links between gaze behaviour and other aspects of socio-cognitive functioning}

The third and final aim of this work was to explore the relationship between sociallyrelevant gaze behaviour and socio-cognitive functioning. The assumption which drives the majority of eye tracking research is that what we look at closely corresponds to what we are thinking about (Yarbus, 1967). As well as exploring where children attend during social interaction (face vs. object; face regions) we also wanted to look at aspects of gaze behaviour that could provide insights into implicit mindreading / mentalising in real-life interactions. Eye tracking techniques are increasingly being used as a method of exploring spontaneous and implicit forms of mentalising, for example, in terms of anticipatory eye movements. Senju, Southgate, White and Frith (2009) have shown how individuals with Asperger syndrome failed to show anticipatory gaze behaviours in line with an actor's false belief even 
though they passed a battery of theory of mind tests. Here, the dissociation between successful performance on verbally mediated false belief tasks (varying in complexity) and 'unsuccessful' performance on the video version of the false belief task provides evidence of an absence of spontaneous/implicit mindreading in autism, even when explicit mindreading is intact. Furthermore, Senju et al. (2010) showed the same absence of spontaneous false belief attribution in children with autism, through a lack of anticipatory gaze which was independent of verbal and non-verbal ability, age, and performance on standard false belief tasks.

False belief tasks, although the gold standard for measuring ToM, are very structured experimental tasks which do not represent the way we mentalise in everyday life (Klin et al., 2002a). Everyday social interaction is unstructured, fluid, and spontaneous and automatic mentalising is necessary for successful social interaction (Senju et al., 2010). Understanding information from the face is a critical part of this because the face contains the information we need to attribute another's focus of attention, mood or emotion, and intention, which allows us to predict other's behaviour (Frith \& Frith, 2006). The use of unexpected events has been important for studying how people use information from faces. An unexpected event creates a situation of uncertainty, and looking at another person's face allows you to check their awareness of what has happened. For example, unexpected events have been used to probe joint attention abilities in children with autism (Mundy, Delgado, Block, Venezia, Hogan \& Seibert, 2003). Other research has used unexpected events in visual illusions to highlight the influence of social cues from faces (Kuhn, Kourkoulou \& Leekam, 2010). Exploring the priority of attention to the face during social interaction when something unexpected happens may reveal aspects of spontaneous mentalising, specifically, the priority for monitoring another's response in terms of their facial expression. 
Therefore, in addition to measuring attention allocation during social interaction, we aimed to capture the attentional response to an unexpected event. We wanted to explore how the social interaction was monitored around the time of this event, and whether the participants' gaze behaviour might indicate 'awareness checking' of the experimenter. This is a novel way of capturing spontaneous real-life mentalising, circumventing the issues traditionally associated with social-cognitive tasks that load heavily on language ability, which may provide insights into importance of anticipatory gaze and the timing of gaze behaviour for understanding others.

\subsection{Current Study}

We have summarised the literature that relates to the three key aims of this research. The first aim is to explore the allocation of attention to socially relevant information during a real life interaction. The second aim relates to the involvement of individuals with SLI as a critical comparison to those functioning on the autism spectrum. While we predicted that TD children and children with SLI would spend similar amounts of time attending the eyes, we predicted that children with autism would spend significantly less time viewing the eyes throughout the interaction. Our predictions for patterns of mouth viewing were exploratory. Increased viewing of the mouth by children with autism only would support the notion of a 'social' compensatory strategy. Increased viewing of the mouth by children with SLI only would support previous reports of a 'communicative’ compensatory strategy to aid audiovisual processing (Hosozawa et al. 2012). Increased viewing of the mouth by both the autism and SLI groups would indicate that this viewing behaviour may be a consequence of developmental delay relating to social/communicative functioning.

The third aim of the work was to explore attention capture by a face versus an object, and to use an unexpected event around the object to explore links between attention and 
socio-cognitive ability in understanding this event. We predicted that the children with autism would show increased attention to the object by comparison to the TD and SLI groups. The unexpected event allowed us to measure the time it took children to fixate back on the face once this had occurred, as an index of anticipatory gaze corresponding to awareness checking. We predicted that children with autism would be slower than the other two groups to make this fixation, and that this behaviour would reflect spontaneous mentalising. Therefore, we predicted that false belief attribution (measure of social-cognitive skill) would correlate with latency, but at the same time, that the latency difference between the groups would be remain even when false belief attribution was co-varied out, showing that this measure reflects a more spontaneous / automatic form of mentalising.

\section{Method}

\subsection{Participants}

All participants were recruited from mainstream or special schools in Northern Ireland. The sample consisted of three groups of children: A group with an ASD; a language comparison group; and a non-verbal ability typically developing comparison group. Thirty children with ASD were recruited, who had previously been diagnosed by experienced clinicians according to the DSM-IV criteria (APA, 1994), and they all had a full statement of special educational needs. Eye-tracking data were obtained from 17 out of 30 children with ASD (reasons for failure to obtain eye tracking data from 13 participants with ASD included difficulties with calibration, inability to comply with task demands, and glasses with anti-

glare coating). The final sample comprised of 17 children with ASD ranging from 7 years and 11 months to 12 years and 11 months (see Table 1 ).

[Table 1] 
A language comparison group of 17 children with a diagnosis of SLI were matched to the ASD group for age and verbal ability using the British Picture Vocabulary Scales II (BPVS II; Dunn, Dunn, Whetton \& Burley, 1997) (Table 1). There was no significant difference between the ASD and SLI groups on the basis of the BPVS raw scores, $t(29)=1.120, p=$ .272 , or age, $t(29)=.811, p=.424$. Children with SLI were recruited from a special school for children with specific language impairments, and each child had a full educational statement detailing their speech and language difficulties. The criterion for entry to the school was a score below the $2^{\text {nd }}$ percentile on overall language ability as measured by the Clinical Evaluation of Language Fundamentals (CELF-4) (Semel, Wiig \& Secord, 2006). Of these 17 participants, eye-tracking data were obtained from 14 , for the same reasons indicated above. Therefore the final sample of children with SLI comprised of 14 individuals ranging from 8 years 1 month to 11 year 1 month (see Table 1 ).

A non-verbal ability comparison group of 21 children who were typically developing were matched to the ASD group for age and non-verbal ability using the Wechsler NonVerbal Scale of Ability (WNV; Wechsler \& Naglieri, 2006). The ASD and TD group did not differ significantly on the basis of chronological age, $t(31)=.214, p=.832$, or on the basis of nonverbal ability, $t(31)=-.386, p=.782$. These children were recruited from a mainstream primary school. Of these 21 participants, eye-tracking data were obtained from 16, for the same reasons indicated above. Therefore the final sample of TD children comprised of 16 individuals ranging from 9 years 1 month to 11 years 9 months (see Table 1).

Ethical approval for this research was granted by the ethics committee in the School of Psychology, Queen’s University Belfast. Parents provided informed consent, and all of the children provided assent prior to taking part. For each child, the experiments were conducted 
individually in a quiet room at their school. Ethical approval was obtained before testing was conducted.

\subsection{Design}

Social interaction by its nature is fluid and unstructured, and therefore a key challenge lay in striking the balance between designing a scenario that was naturalistic yet controlled.

\subsubsection{Real-life interaction and experimental setting.}

Participants took part in a semi-structured interaction with an experimenter posing as a 'magician-in-training' who needed to practise her tricks. The interaction appeared realistic and engaging to the child, but also credible. Children were asked to perform the role of cameraperson, and to record a magician practising her tricks while wearing the eye tracking helmet. There were no tricks involved in the interaction, but a short conversation where the magician introduced herself and asked the participant some questions, followed by a section where the magician introduced a hand puppet and recited a poem about magic (see Semistructured Interaction section).

The experimental setup was designed in such a way as to minimise visual distraction. The participant's chair was positioned 1.5 metres from a blank white wall (this ensured an appropriate visual angle was achieved even for the smallest AOIs, such as the magician's eyes and mouth). The magician sat on a stool with her back to the wall and facing the participant. Two screens with white coverings were placed on the left and right of the experimenter to minimise visual distraction. The computer station for the HED system (see details below) was positioned behind the participant's chair, and was controlled by a second 
experimenter. There were four different female 'magicians', to ensure that effects could not be explained by a response to a particular experimenter ${ }^{2}$.

\subsubsection{Semi-structured interaction.}

Introduction conversation - The purpose of this section was to replicate the everyday scenario of meeting an unfamiliar person for the first time. The magician asked participants the same set of questions, such as, 'what is your name?', 'what age are you' etc., and critically, the participant was required to respond. The magician then spent about 15-20 seconds explaining who she was, and what she was going to do. The introduction conversation lasted 35 seconds on average.

Puppet Section - After the introduction the magician introduced the participant to a wizard puppet, and explained that the wizard was going to tell a magical story. However, there was no ventriloquism involved, and the magician recited the poem while looking straight ahead towards the child and moving the puppet from side to side, adjacent to her face. The purpose of this was to create a scenario where there was another other interesting

\footnotetext{
${ }^{2}$ Although each magician was assigned to test equal numbers of children in each group, because of issues with collecting the eye tracking this was not possible. Analyses showed however, that there was no difference in interaction length between the groups, and that there was no effect of experimenter on the gaze patterns in either part of the interaction. There was no difference in the length of the interaction between the groups, $F(2,44)=$ $1.552, \mathrm{p}>.05$, or for the length of either part of the interaction [Introduction: $F(2,44)=2.452, \mathrm{p}>.05$; Puppet Section: $F(2,44)=.615, \mathrm{p}>.05]$.

Further analyses were carried out to explore the effect of experimenter (A,B,C,D) on gaze patterns. In the introduction section, no effect of experimenter was found on attention to either the whole face, body or nonpartner regions, $\mathrm{F}(3,43)=.594, \mathrm{p}=.623, \eta^{2}=.042$, nor was an interaction effect found between region and experimenter, $F(6,86)=1.259, \mathrm{p}=.285, \eta^{2}=.041$. The same was found for attention to face regions (eyes, nose, mouth, face) in the introduction, as no main effect of experimenter was found $F(3,43)=1.341, \mathrm{p}=.274$, $\eta^{2}=.09$, nor was an interaction found between face region and experimenter, $F(9,129)=.702, p=.707, \eta^{2}=$ .039 .

In the puppet section, no effect of experimenter was found on attention to either the whole face, body or nonpartner regions, $F(3,43)=.746, \mathrm{p}=.530, \eta^{2}=.05$, nor was an interaction effect found between region and experimenter, $F(6,86)=1.042, \mathrm{p}=.404, \eta^{2}=.04$. The same was found for attention to face regions (eyes, nose, mouth, face) in the puppet section, as no main effect of experimenter was found, $F(3,43)=.608, p=.614, \eta^{2}=$ .042 , nor was an interaction found between face region and experimenter, $F(9,129)=1.091, \mathrm{p}=.374, \eta^{2}=.07$.
} 
object competing for their attention besides the magician's face. The poem had 5 verses, each of which was 4 lines long. At the beginning of the fourth verse a second experimenter created an interference by sticking her head out from behind the screen, putting her finger to her lips to indicate that what she was doing was secretive, and pulling the puppet off the magician's hand, letting it drop to the floor. ${ }^{3}$ Critically, the magician continued reciting the poem as if nothing had happened, waving her hand from side to side in the same fashion as before. We were interested in the timing of the first look made back to the magician's face once the puppet had been interfered with (latency). Of course, it is possible that children might look at the face once the interference occurs simply because it is the most interesting thing to look at once the puppet is no longer visible. Therefore, to determine whether our latency measure reflected an anticipation of a response from the magician we explored whether the latency to fixate the face after the interference might be related to a measure of mentalising, namely performance on false belief tasks. Importantly, however, we also wanted to show that our latency measure reflected spontaneous and automatic mentalising and as such we expected that differences between the groups in terms of latency would remain even when false belief attribution abilities were controlled for (as found in Senju et al., 2010). The puppet section (and the interaction) finished when the poem finished.

\subsection{Eye-tracking}

Eye movements were recorded using an SMI Head-mounted Eye-tracking Device (HED; SMI, Germany). The HED allows for eye tracking during real-life interactions. This

\footnotetext{
${ }^{3}$ It was not possible to measure from the eye-tracking videos whether children in the different groups attended to the face of the 'interferer' as described above. As we had instructed children to sit still facing forward, this meant that the scene camera often did not pick up the face of the 'interferer'. Therefore, we could not measure whether children attended to this person's face. We measured 'latency' from the time the interferer's hand was on the puppet, about to pull it off the magician's hand. We used this as the start point for this measure as one might expect this to be the earliest that the magician should have been aware of an interference, through feeling the puppet be tampered with. In terms of where the children were looking at this point, $74 \%$ of the children were looking at the puppet, 17\% classified as 'looking' at the off-screen region, $6.4 \%$ were looking at the body region, and $2 . .12$ were looking at the wall region. We could not conduct chi square analyses on these data between the groups because of the cell count.
} 
system is in the form of a bicycle helmet with a mounted eye camera and scene camera, and weighs 79 grams. Eye tracking was achieved via an infrared eye camera mounted on the helmet, which then overlaid a gaze cursor onto the video recorded by the scene camera, also mounted onto the helmet. The HED sampled at 50-60 Hz, and had a tracking accuracy of between $.5^{\circ}$ to $1^{\circ}$ degrees of the visual angle. Output from this system is in the form of Mpeg video with a gaze cursor overlay.

Calibration for the HED was different to calibration of screen-mounted eye trackers. It involved recording participants' gaze while they fixated on 9 points on the wall which corresponded to known points on the scene video. Participants were directed to fixate on each of the 9 points using a laser pointer. These points subtended a rectangle and corresponded to known locations on the scene video. In order to validate the accuracy of each participant's calibration, we made a large board $(50$ X $60 \mathrm{~cm})$ containing different coloured circles at 9 locations on the board. The participant was asked to look to the different points on the board after calibration to check the accuracy of the calibration. Recording only proceeded on the basis of an accurate calibration.

\subsubsection{Video data coding.}

Videos with gaze cursor overlays were coded on a frame by frame basis using the Observer 5.0 (Noldus, 1994). The videos were coded by two naïve raters blind to the diagnoses of participants. The first rater coded all of the videos, and a second rater coded a percentage of all videos for reliability analyses (see Reliability Analyses). The videos were coded according to the position of the point of regard in relation to specified areas of interest (AOI). AOIs included the magician's eyes, nose, mouth, external face regions (cheeks, forehead, chin), body, hair, hands and hat. There were also AOIs defined for parts of the visual field which did not include the magician. These included the puppet (only visible in the 
puppet section), wall, off-screen, and other. We included the 'off-screen' category to account for blink data and minor data loss. We had one experimenter monitor the eye tracking at all times, and recording was discontinued if the track was lost for more than 5 seconds. However, it became apparent during testing that the off-screen category was in fact a very useful one. We found that there were many times when participants were fixating, but on an area that fell beyond the bounds of the scene camera (i.e. point of regard not visible on the scene video, but not data loss due to poor tracking). We noted that this was particularly pronounced for the children with autism. Therefore, we have used these data in our analyses. The 'other' category reflected instances where the POR fell on the border of two regions, and therefore couldn't be defined as one or the other. For analyses, fixations were defined as $\geq 3$ consecutive frames $(120 \mathrm{~ms})$ of stable gaze on an AOI, following the conventions of previous studies using head-mounted eye-tracking (Franchak, Kretch, Soska \& Adolph, in press).

\subsubsection{Reliability analyses.}

Twenty percent of each video was coded by a second rater, and Cohen's Kappa coefficient and percentage of agreements were calculated using Observer to assess inter-rater reliability. These analyses were based on the duration and sequence of agreements. The average $\kappa$ was 0.84 , and the average percentage of agreement was $88 \%$ indicating excellent agreement.

Test re-test reliability was also assessed as the first rater re-coded a full video from one participant two weeks after the first coding, and again, Kappa Co-efficients and percentage of agreement indicated excellent test re-test reliability $(\kappa=.79$; agreement $=$ $81 \%)$.

\subsection{Socio-cognitive tasks}


Participants completed a battery of Theory of Mind (ToM) tests in a separate testing session. Our battery of four false belief tasks included: Unexpected Contents First-Order False-belief (Smarties Task; Wimmer \& Hartl, 1991); Unexpected Location First-Order False-belief (Sally and Andy Task; Wimmer \& Perner, 1983); Belief-Desire Reasoning (Nice Surprise Task; Harris, Johnson, Hutton, Andrews \& Cooke, 1989); and Unexpected Location Second-Order False-belief (Grandad Story; Perner \& Wimmer, 1985). The tasks were taken from Hughes, Adlam, Happé, Jackson, Taylor and Caspi (2000), and we followed the procedure of Paynter and Peterson (2010).

Each task had one or two control questions and one ToM test question. To pass each test question, children were also required to respond correctly to the accompanying reality control question. All children answered the reality control questions correctly. In total, there were four standard ToM questions and two advanced ToM questions, where each correct response was awarded one mark. Standard theory of mind questions, requiring participants to form a prediction based on a false belief, featured in the Smarties Task and the Sally-Andy task. In the instance where a participant failed both first order belief tasks, testing was discontinued. Five children with autism and one child with SLI failed both first order test questions. Advanced ToM questions examined the ability to infer what a character thinks another character is thinking, such as in the Grandad story. The ToM question in the beliefdesire reasoning task (nice surprise) required participants to infer an emotion from an attributed false belief. A total composite score (maximum of 6) was then calculated for each participant.

\subsection{Procedure}


All participants were tested individually in a quiet room in their schools. They all completed the eye-tracking session first, and only completed the ToM testing session if eyetracking data had been successful recorded. Once the participant was seated in a comfortable chair, they were shown the scene camera on the eye-tracking helmet and how it worked. Calibration of the eye-tracker was then carried out. Once an accurate calibration was achieved, the magician sat down on the stool facing the participant and initiated the semistructured conversation. Once this was completed the participant was thanked for their help. All children who had completed the eye-tracking tasks successfully completed the false belief task battery in a separate testing session.

\section{Results}

The data were considered first for viewing patterns during the introduction conversation, and secondly for the puppet section (content and AOIs differed between sections). Analyses of the gaze data explored group differences in percentage of total fixations made to AOIs. For each section, gaze behaviour was first considered towards the whole face, body and non-partner areas and then in terms of face regions (see Figures 1a,b,\& c). A further analysis was conducted for the puppet section to explore attention capture between the magician's face and the puppet. The final sections of the results concern latency and false belief task performance. ${ }^{4}$

\footnotetext{
${ }^{4}$ In research involving clinical groups, extreme values are often clinically and theoretically significant. ANOVA is also a particularly robust statistical method. Nonetheless, all analyses were checked for outliers 2 standard deviations above the mean, and analyses were re-run with any outliers removed. There were never more than three outliers per variable, and removal of outliers did not change the outcome of any of the analyses. Therefore, for consistency outliers were not removed from the analyses presented in this paper.
} 


\subsection{Introduction Conversation - Attention Patterns}

Eye-tracking data are expressed as a percentage of total fixations made within the introduction section. However, data from the AOIs hat, hair, hands and 'other' were not included in these analyses, and as such no individual participant's data equalled 100 per cent ${ }^{5}$.

[Figure 1]

\subsubsection{Face (whole), body \& non-partner (wall, off-screen) regions.}

Figure 1a shows the percentage of fixations to the AOIs across the groups and it is possible to see considerable differences between the ASD group and the two comparison groups in terms of looking to the whole face and the non-partner areas. An ANOVA with factors group (ASD, SLI, TD) and region (whole face, body, non-partner areas) was carried out on the gaze data from the introduction section. Although there was no main effect of group, $F(2,44)=2.324, p=.110$, there was a significant main effect of region, $F(2,88)=$ 70.65, $p<.001$, and a critical interaction between the factors, $F(4,88)=11.736, p<.001$.

The region whole face was looked at for longer than both the body region, $t(46)=$ $10.914, p<.001$, and the non-partner regions, $t(46)=3.640, p<.01$. The non-partner regions were looked at for longer than the body region, $t(46)=7.411, p<.001$.

In terms of the interaction between group and region, it was found that the groups differed for the way they viewed the whole face, $F(2,44)=15.772, p<.001, \eta^{2}=.417$, the

\footnotetext{
${ }^{5}$ The summed percentage of data (whole face, body and non-partner areas) for individual participants ranged from $63 \%$ to $99 \%$ with a mean of $83.2 \%$ and a standard deviation of $9.9 \%$.
} 
body, $F(2,44)=3.534, p=.038, \eta^{2}=.138$, and the non-partner areas, $F(2,44)=7.420, p=$ $.002, \eta^{2}=.252$. Tukey post-hoc tests revealed that the ASD group viewed the whole face for a significantly smaller percentage (e.g. for shorter time) than both comparison groups (ASD-

SLI: $p<.001$; ASD - TD: $p<.001$ ) and the non-partner regions for a significantly longer time (a higher percentage of viewing time) than both comparison groups (ASD-SLI: $p=.008$; ASD - TD: $p=.004)$. It must be noted that increased looking to the non-partner regions by the ASD group was mainly because of increased looking off-screen (Mean ASD: non-partner = 39.96; off-screen $=35.74$; wall $=4.21$ ) although the ASD group did also look longer to the wall than the other two comparison groups. The SLI and TD groups did not differ in the way they viewed any of these regions (face: $p=.888$; non-partner: $p=.992$ ).

The effect for viewing of the body was driven by the ASD group spending a longer percentage of time viewing the body in comparison to the SLI group $(p=.041)$ but there were no differences between the ASD and TD group $(p=.138)$ or the SLI and TD groups ( $p$ $=.806)$.

\subsubsection{Face regions (eyes, nose, mouth, external face region).}

Inspection of Figure 1b indicates a substantial difference between the ASD group and the two comparison groups in terms of the percentage of fixations to the eye region. To explore the distribution of attention to face regions between the groups an ANOVA with factors group (ASD, SLI, TD) and face region (eyes, nose, mouth, external face) was carried out. There was a significant main effect of group, $F(2,44)=15.749, p<.001$, a significant main effect of face region, $F(3,132)=11.115, p<.001$, and a significant interaction of group and face region, $F(6,132)=4.247, p=.001$. 
The eyes were viewed for longer than the nose and the external face regions (eyesnose: $t(46)=4.109, \mathrm{p}=<.001$; eyes-face external; $t(46)=4.292, \mathrm{p}<.001)$. The eyes were viewed longer than the mouth region, although this was a trend towards significance, $t(46)=$ $1.814, \mathrm{p}=.076$. The nose was viewed for a similar percentage of time in comparison to the mouth and the external face regions (nose-mouth: $t(46)=-1.807, \mathrm{p}=.077$; nose-face: $t(46)$ $=.803, \mathrm{p}=.426)$. Finally, the mouth was viewed for longer than the external face regions, $t$ $(46)=3.082, p=.003$.

Groups differed in the percentage of time spent viewing the eyes, $F(2,44)=12.185$, $p$ $<.001, \eta^{2}=.352$, and the nose, $F(2,44)=5.478, p=.008, \eta^{2}=.199$, but not the mouth, $F(2$, $44)=.146, p=.864, \eta^{2}=.006$, or the external face, $\mathrm{F}(2,44)=.544, p=.584, \eta^{2}=.024$.

Tukey post-hoc tests showed that the ASD group spent a significantly shorter portion of time viewing (smaller percentage) the eyes than both comparison groups (ASD-SLI: $p=.001$; ASD-TD: $p<.001$ ) although there was no difference between the SLI and TD groups (SLITD: $p=.820$ ). The group difference for viewing the nose was driven by the TD group spending a greater percentage of time viewing the nose than the ASD group (TD-ASD: $p=$ .005) whereas there was no difference between the TD and SLI groups $(p=.330)$ or the ASD and SLI groups $(p=.206)$.

\subsection{Puppet Section - Attention Patterns}

The puppet section of the interaction had two parts to it separated by the interference - in the first part the puppet was visible; and the second part after the interference, the magician's hand was visible waving side to side where the puppet had been. For the gaze data from the puppet section, we first consider attention allocation to the same AOIs as in the introduction section, i.e. face, body and non-partner regions first, followed by an analysis of face regions for the whole puppet section (data from figure $1 \mathrm{~b}$ and 1c combined). Therefore, 
data from the AOIs puppet/hand, hat, hair, hands and 'other' were not included in these analyses, and as such no participant's data equalled 100\%. ${ }^{6}$ An analysis of attention capture between the puppet and the face is conducted separately.

\subsubsection{Face (whole), body \& non-partner (wall, off-screen) regions.}

Looking at Figure 1, it is possible to see that the introduction of the puppet influenced the allocation of attention to the other regions. However, aside from attention to the puppet (which is analysed separately) gaze patterns were largely similar to the introduction, with the ASD group spending less time looking at the whole face than the SLI and TD groups, and more time at the body and non-partner areas. An ANOVA with factors group (ASD, SLI, TD) and region (whole face, body, non-partner areas) was conducted on the gaze data from the puppet section. Although there was no main effect of group, $F(2,44)=.206, p=.814$, there was a significant main effect of region, $F(2,88)=44.316, p<.001$, and a significant interaction between the factors group and region, $F(4,88)=8.587, p<.001$. The whole face region was attended to for longer than the body region, $t(46)=7.731, \mathrm{p}<.001$, and the nonpartner regions, $t(46)=3.599, \mathrm{p}<.01$. The non-partner regions were viewed for longer than the body region, $t(46)=6.967, \mathrm{p}<.001$.

In terms of the interaction between group and region, groups differed in the way they viewed the whole face, $F(2,44)=5.853, p=.006, \eta^{2}=.194$, the body, $F(2,44)=8.980, p$ $=.001, \eta^{2}=.289$, and the non-partner areas, $F(2,44)=9.268, p<.001, \eta^{2}=.296$. Tukey post-hoc tests revealed that group differences for viewing of the whole face were driven by the ASD group looking less at the whole face than both comparison groups (ASD-SLI: $\mathrm{p}=$ .014; ASD-TD: $\mathrm{p}=.015$ ), whereas there was no difference between the SLI and TD groups $(p=.989)$. Differences for viewing the body region were driven by the ASD group spending

\footnotetext{
${ }^{6}$ The summed percentage of data (whole face, body and non-partner areas) for individual participants ranged from $9.72 \%$ to $99.2 \%$ with a mean of $53.36 \%$ and a standard deviation of $21.7 \%$.
} 
significantly longer looking to the body than both comparison groups (ASD-SLI: $\mathrm{p}=.004$; ASD-TD: $\mathrm{p}=.001)$, whereas there was no difference between the SLI and TD groups $(p=$ .961). Similarly, group differences for viewing the non-partner areas were driven by the ASD group also spending a greater percentage of time looking at these regions than both comparison groups (ASD-SLI: $p=.008$; ASD-TD: $p=.001$ ). Again, the SLI and TD groups did not differ for looking to the non-partner regions $(p=.724)$. It must be noted that increased looking to the non-partner regions by the ASD group was mainly because of increased looking off-screen (Mean ASD: non-partner = 26.43; off-screen = 23.32; wall = 3.1) although the ASD group did also look longer to the wall than the other two comparison groups.

\subsubsection{Face regions (eyes, nose, mouth, external face region).}

Again, Figure 1 (a, b, c) shows the influence that the puppet had in terms of overall percentage of attention allocated to the face regions. However, the pattern observed in the introduction is very similar here, with the ASD group spending much less time looking at the eyes than the other two comparison groups. To explore the distribution of attention to the face during the puppet section an ANOVA with factors group (ASD, SLI, TD) and face region (eyes, nose mouth, external face) was conducted. There was a main effect of group, $F(2,44)$ $=6.087, p=.005$, main effect of face region, $F(3,132)=7.421, p<.001$, and an interaction effect of group and face region, $F(6,132)=4.646, p<.001$.

The eyes were looked at for longer than all other face regions (eyes-nose: $t$ (46) $=$ 2.556, $\mathrm{p}=.014$; eyes-mouth: $t(46)=3.026, \mathrm{p}=.0004$; eyes-face external; $t(46)=3.099, \mathrm{p}=$ .003). However, there were no differences between the other face regions for percentage of looking (nose-mouth; $t(46)=.138, \mathrm{p}=.891$; nose-face external: $t(46)=.440, \mathrm{p}=.662$; mouth-face external: $t(46)=.409, \mathrm{p}=.684)$. 
The interaction effect was driven by the way the groups viewed the eyes, $F(2,44)=$ 9.536, $p<.001, \eta^{2}=.301$, but not the mouth, $F(2,44)=2.504, p=.093, \eta^{2}=.102$, the nose, $F(2,44)=.956, p=.392, \eta^{2}=.041$, or the external face region, $F(2,44)=.426, p=.655, \eta^{2}$ $=.019$. Tukey post-hoc tests revealed that the ASD group viewed the eyes for less time than both comparison group (ASD-SLI: $p=.005$; ASD-TD: $p=.001$ ). The SLI and TD groups did not differ for time spent viewing the eyes $(p=.805)$.

\subsection{Summary of Gaze Behaviour during Introduction and Puppet Sections}

During both sections of the interaction, the whole face was viewed for longer than any other region. The body region was viewed for the least amount of time, and a considerable amount of time was classified as off-screen, in both sections. The pattern of attention allocation differed between the groups however, with the ASD diverging significantly from both the SLI and TD groups in terms of the amount of time spent viewing the whole face in both sections, the non-partner areas in both sections, and the body in the puppet section. The profile of attention by the ASD group was one of less time spent looking at the face and more time looking at the body, wall and off-screen regions.

This pattern of reduced social interest by the ASD participants was also observed in the way they allocated their attention to the face regions. Although the eyes were looked at for longer than the other face regions overall, this patterns was not reflected at the group level. The ASD group spent significantly less time viewing the eyes than both the SLI and TD groups in both sections.

\subsection{Attention Capture between the Puppet and the Face - Before and After the Interference}

\subsubsection{Puppet present.}


In order to explore how attention was distributed between the puppet and the experimenter's face during the puppet section, an ANOVA with factors group (ASD, SLI, TD) and region (puppet, face) was conducted on the data during which the puppet was present. There was a significant main effect of group, $F(2,44)=13.353, p<.001$, and a significant main effect of region, $F(1,44)=30.763, p<.001$, but the interaction between the factors was not significant, $F(2,44)=.841, p=.438$. In terms of the main effect of group, we carried out t-tests which showed that the ASD group spent less time looking to both the puppet and whole face regions than both comparison groups (ASD - SLI: $t(29)=-3.228, p<$ .01 ; ASD - TD: $t(31)=4.356, p<.001$; TD - SLI; $t(28)=1.716, p=.097)$. In terms of the effect of region, all participants spent a greater percentage of time viewing the puppet than the face, $t(46)=5.689, \mathrm{p}<.001, \mathrm{r}=-.775$.

We also conducted paired $t$ tests within groups to explore whether each group was equally likely to look at the puppet and the whole face. The ASD and TD groups looked at the puppet significantly more than the face (ASD: $t(16)=5.757, p<.001$; TD: $t(15)=$ 4.051, $p=.001)$. Although the SLI looked longer at the puppet than the face, this was not significant, $t(13)=1.493, p=.159$.

\section{4.. Puppet gone.}

Attention capture was also explored after the puppet had disappeared and only the experimenter's hand was visible, using an ANOVA with factors group (ASD, SLI, TD) and region (hand, face). There was a significant main effect of group, $F(2,44)=12.467, p<.001$, a significant main effect or region, $F(1,44)=11.785, p=.001$, but the interaction between the factors was not significant, $F(2,44)=2.027, p=.144$. In terms of the main effect of group, we carried out t-tests which showed that the ASD group spent less time looking to both the hand and whole face regions than the comparison groups (ASD - SLI: $t(29)=$ - 
3.291, $p$ <.01; ASD - TD: $t(31)=4.572, p<.001$; TD - SLI; $t(28)=.684, p=.500)$. In terms of the effect of region, all participants spent a larger percentage of time viewing the face than the hand, $t$ (46) $=-3.261, p=.002, r=-.568$.

We conducted paired $t$ tests within groups to explore whether each group was equally likely to look at the hand and the whole face. The SLI and TD groups looked at the face significantly more than the hand (SLI: $t(13)=-2.192, p=.047$; TD: $\mathrm{t}(15)=-2.698, p=$ .017). The ASD group were equally likely to look at the hand or the face, $\mathrm{t}(16)=-.535, p=$ 600 .

\subsubsection{Latency to look back at experimenter's face after interference.}

A measure of anticipatory gaze was taken as the time (in seconds) for participants to look back at the experimenter's face once the puppet has been interfered with (see Figure 2). A univariate ANOVA with factor group (ASD, SLI, TD) on latency to look back the experimenter's face was conducted. A main effect of group was significant, $F(2,44)=8.932$, $p=.001, \eta^{2}=.29$, and Tukey post-hoc tests revealed that the ASD participants had greater latencies (and were therefore slower to look back at the experimenter's face) than both comparison groups (ASD-SLI: $p=.01$; ASD-TD: $p=.001$ ), whereas there was no difference between the TD and SLI groups $(p=.723)$.

\section{[Figure 2]}

Furthermore, we explored the region of the face to which the groups made their first fixation. 5.9\% of the ASD group made their first fixation to the eyes, compared to $35.7 \%$ of the SLI group, and $37.5 \%$ of the TD group. $11.76 \%$ of the ASD made their first fixation to the nose, compared with none from the SLI or TD groups. $41.2 \%$ of the ASD group made their first fixation to the mouth compared with $35.7 \%$ of the SLI group and $6.25 \%$ of the TD 
group. Finally, $41.2 \%$ of the ASD group made their first fixation to the external face region, compared with $28.6 \%$ of the SLI group and $56.25 \%$ of the TD group. However, due to insufficient cell count, we could not conduct the chi square on the data for face region. Therefore, we re-categorised the data in terms of upper or lower face region, with the dividing point between upper and lower being the tip of the nose. There was a very strong statistically significant association between group and the region of the face fixated first $\chi^{2}$ $(2,47)=16.228, p<.001 ; V=.59$. A much greater proportion of the autism group, $82.4 \%$, fixated on the lower region of face relative to the SLI group (42.9\%; odds ratio 6.2) or the TD group (12.5\%; odds ratio 32.7$)$.

\subsection{Relationship between Social Cognition and Social Attention}

\subsubsection{Theory of mind performance.}

Results from the composite scores of the ToM battery showed that the TD group had the highest composite scores, followed by the SLI group, with the ASD group showing the lowest composite scores (see Figure 3). A one-factor univariate ANOVA confirmed that there was a significant main effect of Group on ToM performance, $F(2,44)=14.598, p<.00, \eta^{2}$ $=.39$. Tukey post-hoc tests revealed that the TD group performed significantly better than the SLI group $(p<.05)$ and the ASD group $(p<.001)$, while the SLI group performed significantly better than the ASD group $(p<.05)$.

\section{[Figure 3]}

These effects remained when BPVS raw score and age in months were co-varied out between the TD and the ASD group, and the TD group and the SLI group (ASD: TD - F(1, 29) = 36.307, $p<.001, \eta^{2}=.50$; SLI: TD $\left.-F(1,26)=6.578, p<.05, \eta^{2}=.201\right)$. However, the effect 
did not remain for the difference between the ASD and SLI groups [ASD: SLI - $F(1,27)=$ 3.502, $\left.p=.072, \eta^{2}=.108\right]$.

A series of correlations were conducted to explore the relationship between aspects of gaze behaviour and performance on the ToM battery (composite score), while controlling for age in months and BPVS raw scores. To avoid confounds of small sample size and to provide sufficient statistical power, the full sample, across groups, is used for these correlations. Although not relevant to the precise research question set out here, Table 2 provides additional correlation data relating to task performance (BPVS, ToM, age) and gaze behaviour.

\section{[Table 2]}

\subsubsection{Looking to the eyes and ToM.}

The percentage of time spent looking at the eyes throughout the interaction was found to be associated with ToM performance, $r(43)=.240, p=.056$, such that more time spent attending to the eyes of the magician was associated with better ToM performance when age and BPVS raw score were controlled for, although this result only approached statistical significance.

\subsubsection{Anticipatory gaze (awareness checking) and ToM.}

The relationship between the time it took for participants to look back at the experimenter's face once the puppet had been interfered with (latency) and ToM performance was explored. Anticipatory gaze (latency) and ToM composite scores were found to be significantly associated, $r(43)=-.312, p=.018$, when age and BPVS raw scores were partialled out, such that greater latencies (the slower participants were to look back at the experimenter’s face) were related to poorer ToM performance. 
Furthermore, in terms of our anticipatory gaze measure it was found that pattern previously observed between the groups (greater latencies by the ASD group in comparison to the SLI and TD groups) remained when ToM composite score was co-varied out (ASD: $\mathrm{TD}-F(1,230)=5.136, p<.05, \eta^{2}=.12$; ASD: SLI $-F(1,28)=5.895, p<.05, \eta^{2}=.16$; SLI: $\left.\mathrm{TD}-F(1,27)=.868, p<.05, \eta^{2}=.03\right)$.

\section{Discussion}

This study explored social gaze (a)typicality in autism and its relation to sociocognitive ability during a real interaction. Individuals with ASD showed syndrome-specific atypicalities of social attention allocation that dissociated them from those with SLI and TD children. When faced with a natural person-to-person interaction, individuals functioning on the autism spectrum showed atypically reduced face gaze, especially to the eye region. We discuss the findings in terms of each of the aims of the study and the predictions made in relation to those aims. We then pull the findings together to consider the overall clinical implications.

\subsection{Attention to the face during real interaction}

Despite their obvious developmental differences (language ability), we found the gaze behaviour of TD and SLI children to be very 'social'. The children in both these groups prioritised information from the interlocutor's face. Children with autism on the other hand looked significantly less to the face, and spent a significantly larger proportion of time looking to the body region and non-partner regions. This supports previous studies indicating reduced face gaze in autism (e.g. Riby \& Hancock, 2009b). However, we provide the first eye 
tracking evidence of reduced face gaze during real interaction, contrasting with the findings of Nadig et al. (2010). Importantly, we also provide evidence of attention to face regions and indicate that not only is face gaze reduced in autism, but attention to the eyes of the interlocutor is most atypical, which has theoretical significance (Klin \& Jones, 2008; Pruett et al., 2011; Senju \& Johnson, 2009). This supports previous screen-viewing eye-tracking studies (Hanley et al., 2012; Klin et al., 2002b).

Our findings in terms of reduced face gaze in autism contrast with the only other published study using eye tracking during real interaction with children with the disorder (Nadig et al., 2010). Whereas Nadig et al. (2010) indicated children with autism showed typical gaze behaviour while mostly speaking, our study showed atypical gaze behaviour while children with autism were mostly listening. It is possible that the differences between the studies are due to methodological variations with task demands (speaking vs. listening; for example see Doherty-Sneddon et al., 2012 work on gaze during interaction phases in ASD). It is worth noting that the current study provides the first empirical eye tracking evidence to support gaze behaviour studies that suggest decreased face gaze when children with autism are listening as part of a real life person-to-person interaction (Doherty-Sneddon et al., 2012, 2013). A lack of face gaze when listening will inhibit the ability to detect and utilise non-verbal face cues that aid interpersonal communication.

\subsection{Social Gaze in ASD compared to SLI}

As mentioned above, patterns of attention for children with SLI diverged from autism, particularly in terms of face gaze and eye gaze. However, we also wanted to examine a possible attention bias towards the mouth, given previous research indicating that this bias might represent a compensatory strategy (Hosozawa et al., 2012; Klin et al., 2002b). We expected that if this compensatory strategy existed (for either social or communicative 
reasons), it would be most likely to occur during a real social interaction. Although we found a tendency for the children with SLI to look slightly longer at the mouth throughout the interaction than the other two groups, there were no group differences in any part of the interaction.

Identification of attentional compensatory strategies has the potential to inform intervention, and although we do not report evidence of a mouth-based attentional bias, we do note that our sample sizes were small. It is possible for example, that a mouth-bias characterises some people with a developmental condition, but not all capturing withinsyndrome heterogeneity. For example, there were four participants across the two developmental disorder groups that showed very high levels of mouth looking by comparison to all other participants in the introduction section of the interaction (Mean gaze to mouth for all participants $=13 \%$, SD $=14 \%$; Participant examples: ASD $1=39.01 \%$, ASD $2=53.90 \%$; SLI $1=44.11 \%$, SLI $2=65.70 \%$ ). While we are not suggesting that this is evidence of a mouth bias, it highlights the point that considerable individual differences exist, and that more research is needed to explore this important issue of compensatory strategies, but with larger participant samples to allow for more sophisticated analyses (e.g. cluster analyses). Indeed exploring within-syndrome heterogeneity and developmental change in attention strategies remains a challenge for future research.

Finally, while Hosozawa et al., 2012 was the only study known to the authors comparing autism and SLI on attention to social stimuli, we note that Kelly, Walker and Frazier Norbury (2013) made this cross syndrome comparison while looking at oculomotor control. They found that children with language impairment and children with autism who also had language impairment showed very similar difficulties on tasks measuring volitional oculomotor control, as opposed to children with autism with normal language, and children 
who were developing typically. While it is difficult to extrapolate their findings to the current study (they used non-social stimuli and different indices of attention), their study emphasises the need for more comparisons of SLI and autism, and also the need for studies to include multiple measures of attention beyond total looking times to AOIs to inform our understanding of neurodevelopmental disorders.

\subsection{Looking Time in Non-partner Areas}

Our participants with autism spent a significantly greater percentage of time throughout the interaction looking at the non-partner areas than both comparison groups. Although previous studies have demonstrated increased gaze aversion when children with ASD are listening to an interlocutor (using video techniques; Doherty-Sneddon et al., 2012; 2013), we propose that the increased looking time in the non-partner category in autism may represent evidence of gaze aversion. This category included looking at the background wall region and also time when the point of regard was not visible on the eye-tracking videos (coded as off-screen). We suggest that the off-screen category largely represented time spent looking ‘away' from the interaction when participants were fixating beyond the bounds of the scene camera. There are other reasons for off-screen data, such as blinks or data loss, but we have no a-priori reason to suggest that these should occur more for the autism group then for the other two groups. This is potentially important, given the amount of looking time that was categorised as non-partner (and particularly off-screen) for the children with autism (especially in relation to the comparison groups). On average, children with autism had more looking time in this category compared to all other categories (always more than face). This is not something that has been reported in previous eye-tracking studies on autism, and possibly reflects the demands of real social interaction as opposed to watching social interaction on a screen. For example, it is possible that such looking behaviour could be 
driven by distraction from aspects of the environment or a lack of interest. Alternatively, it is possible that social interaction results in increased physiological arousal for children with autism, and looking away is a compensatory strategy to manage increased arousal (Riby, Whittle \& Doherty-Sneddon, 2012). This is an issue for further investigation. It is noteworthy that for the children with autism, the only period in the interaction where nonpartner gaze was not the most attended category was when the puppet was introduced into the interaction.

\subsection{Attention Capture - Face vs. Object}

As well as exploring attention allocation during interaction, we aimed to explore how attention was captured when an interesting object was introduced. Attention capture between faces and inanimate objects is something that has been considered previously in eye-tracking research, and children with autism have been found to give attentional preference to objects than people (Klin et al., 2002; Sasson et al., 2008). We present the first eye-tracking data on attention capture between objects and faces in real interaction. When the interlocutor introduced the puppet, we predicted that the children with autism would pay much more attention to it than the other two comparison groups. However, this was not the case, as all children found the puppet equally engaging and looked at it significantly more than the magician's face. This was particularly interesting in light of the influence that the introduction of the puppet had on the attention of the children with autism - the introduction of the puppet was the only time in the interaction when looking time for the children with autism was greatest in a region other than the non-partner regions. When the puppet was removed and only the magician's hand was visible, the children with autism returned to a higher percentage of looking time in the non-partner areas than any other region. Not only does this have implications for understanding how to engage children with autism, but it 
further highlights the issue of the possible function of increased looking in the non-partner category. The children with autism could re-orient their attention in the direction of the interlocutor when an interesting object was introduced.

When we looked at gaze behaviour after the puppet was removed, the overall pattern of attention had changed considerably, such that the interlocutor's face was looked at for longer than her hand. However, this was largely driven by the two comparison groups, who spent significantly more time looking at the magician's face than her hand, whereas the children with autism were equally likely to look at either region.

This was a novel way of probing face versus object attention capture, which has interesting implications for engaging children with autism. All of the children were equally interested in the puppet, but the appearance of the puppet had a different impact on attention allocation for the children with autism. The appearance of the puppet drew the attention of the children with autism back towards the interaction and engaged their attention for as long as it was visible. A commonly held view is that children with autism show a maladaptive interest / preference for inanimate objects (Celani, 2002), but our findings highlight the potential for using teaching aids such as hand puppets to aid engagement and learning for children with autism, and this is something that should be investigated in future work.

\subsection{Social Gaze and Links to Socio-Cognitive Abilities}

The final novel aim of this study was to explore links between social gaze and sociocognitive abilities. It has been suggested that eye tracking during real interaction may provide an ideal way of probing spontaneous and implicit mentalising (Skarrat, Cole, \& Kuhn, 2012). We provide the first evidence of this by comparing the performance of children with autism, SLI and TD children on traditional measures of social cognition (ToM) to performance on a real life gaze measure of awareness checking. Our results indicated that children with autism 
and SLI had a deficit in explicit mentalising based on their performance on a false belief test battery. This is line with previous studies of autism (Baron-Cohen et al., 1995; Senju et al., 2010) and SLI (Farrant, Fletcher \& Mayberry, 2006). Interestingly however, there was a very different pattern of performance on our awareness checking measure across the three groups. Here, children with autism were significantly slower than both children with SLI and TD children to look at the interlocutor's face after a trick had been played. Children with SLI and TD children were much quicker to check the face of the interlocutor for signs that she was aware of what had happened (an important behaviour for social monitoring, facilitating the understanding of another's perspective in interaction). Furthermore, our results indicated that our awareness checking measure was related to mentalising ability, but group differences remained in awareness checking even when explicit mindreading was taken in to account. Finally, the proportion of time spent looking at the eyes during the interaction was related to explicit mentalising ability.

This type of evidence may provide an implicit indicator of mentalising ability, above and beyond the skills shown on verbally-mediated tasks. The real life use of this type of information should be explored in future research but here we emphasise with preliminary evidence that eye tracking could be useful in exploring the relationship between attentional strategies during real interaction and other socio cognitive capacities that impact upon everyday behaviour. This type of information is essential for extrapolating basic experimental work to use in more applied interventions and for understanding the everyday needs and abilities of individuals with autism, and indeed other disorders of development such as SLI. These findings emphasise how valuable it is to go beyond total looking times in eye-tracking work, and consider the time-course of gaze behaviour. Freeth et al. (2010) and FletcherWatson et al., (2009) found subtle differences in the timing of gaze behaviour between high functioning adults with ASD and TD controls, even though there were no total looking times 
differences report to regions of interest (e.g. face, eyes). Here again we show the importance of examining the timing of gaze behaviour, and future work should involve more detailed analyses of the timing of gaze for revealing important aspects of online processing of information during real social-interaction.

\subsection{Conclusions, Implications and Future Challenges}

Overall, the findings described above indicate a clear divergence in the way in which children with autism attend to people and faces during social interaction in comparison to TD children and importantly, in comparison to children with SLI. Even though children with SLI experience similar communication difficulties to children with autism which impact on their socio-communicative functioning, their attention patterns are altogether more 'social', which probably aids their social skills. Furthermore, the atypicalities of attention to faces, especially the eye region, shown by individuals with autism are likely to have far reaching consequences, as shown by the use of a real life person-to-person interaction in the current study. We need to consider atypical attention strategies of individuals with autism more carefully when they are listening during a person-to-person interaction. It may be at this point when we should implement appropriate intervention strategies as it is critical that intervention programmes understand the need for gaze behaviour to change as a function of phases of an interaction (e.g. when listening, thinking or speaking, see Doherty-Sneddon et al., 2012; Riby et al., 2012).

Critically, in the current research we have shown that not only are the attention patterns of children with autism not typical when they attend during a real person-to-person interaction, but neither are they due to having a developmental condition impacting on sociocommunicative functioning. We would therefore propose that atypically reduced attention allocation to faces, especially the eye region, during a person-to-person interaction is 
syndrome specific in relation to autism and this will have important implications for future research and in the longer term interventions aimed at children with developmental disorders. We have also shown the utility of real life interaction paradigms using eye tracking for probing real life mentalising. This timely and novel study shows the opportunities available for future research utilising eye tracking methods, involving a real person-to-person interaction and linking to wider issues of social cognition and everyday functioning. 
Acknowledgements

We are grateful for the kind cooperation of the participants involved in this study, their parents and schools. We acknowledge the support of Belvoir Park Primary School, Harberton Primary School, Thornfield Primary School, and St. Kieran’s Primary School. We also acknowledge the technical guidance provided by Dr Martin Sawey, School of Psychology, Queen’s University Belfast. 


\section{References}

American Psychiatric Association. (1994). Diagnostic and statistical manual of mental disorders, DSM-IV (4 $4^{\text {th }}$ ed.). Washington, DC: Author.

American Psychiatric Association (2000). Diagnosis and Statistical Manual of Mental Disorder (DSM-IV-TR) (4 ${ }^{\text {th }}$ Ed.). Washington DC. American Psychiatric Association.

Bar-Haim, Y, Shulman, C., Lamy, D. \& Reuveni, A. (2006). Attention to the Eyes and Mouth in High-Functioning Children with Autism. Journal of Autism and Developmental Disorders, 36, 131-137.

Bellini, S., Peters, J., Benner, L., \& Hopf, A. (2007). A meta-analysis of school-based social skill interventions for children with autism spectrum disorders. Remedial and Special Education, 28, 153- 162.

Bishop, D. V. M. \& Norbury, C. F. (2005). Executive functions in children with communication impairments, in relation to autistic symptomatology: I. Generativity. Autism, 9, 7-27.

Boraston, Z., \& Blakemore, S.J. (2007). The application of eye-tracking in the study of autism. Journal of Physiology, 581, 893-898.

Brock, J., Norbury, C., Einav, S. \& Nation, K. (2008). Do individuals with autism process words in context? Evidence from language-mediated eye-movements. Cognition, 108, 896-904.

Burack, J. A., Iarocci, G., Flanagan, T., \& Bowler, D. (2004). On mosaics and melting pots: Conceptual considerations of comparison and matching strategies. Journal of Autism and Developmental Disorders, 34, 65-73. 
Celani, G. (2002). Human beings, animals and inanimate objects. What do people with autism like? Autism, 6, 93-102.

Corden, B., Chilvers, R., \& Skuse, D. (2008). Avoidance of emotionally arousing stimuli predicts social-perceptual impairment in Asperger’s syndrome. Neuropsychologia, 46, 137-147.

Dawson, G., Meltzoff, A.N., Osterling, J., Rinaldi, J. \& Brown .E. (1998). Children with Autism Fail to Orient to Naturally Occurring Social Stimuli. Journal of Autism and Developmental Disorders, 28, 479-485.

Dawson, G. Toth, K., Abbott, R., Osterling, J., Munson, J., Estes, A. \& Liaw, J. (2004). Early Social Attention Impairments in Autism: Social Orienting, Joint Attention, and Attention to Distress. Developmental Psychology, 40, 271-283.

Dunn, L., Dunn, L., Whetton, C., \& Burley, J. (1997). British Picture Vocabulary Scale II. London: GL Assessment.

Doherty-Sneddon, G., Riby, D.M., \& Whittle, L. (2012) .Gaze aversion as a cognitive load management strategy in autism spectrum disorder and Williams syndrome. Journal of Child Psychology and Psychiatry, 53(4), 420-430.

Doherty-Sneddon, G., Whittle, L. \& Riby, D.M. (2013). Gaze aversion during social style interactions in autism spectrum disorder and Williams syndrome. Research in Developmental Disabilities, 34, 616-626.

Falck-Ytter, T., Fernell, E., Gillberg, C. \& von Hofsten, C. (2010). Face scanning distinguishes social from communication impairments in autism. Developmental Science, 13, 864-875. 
Farrant, B.M., Fletcher, J.F. \& Maybery, M.T. (2006). Specific Language Impairment, Theory of Mind, and Visual Perspective Taking: Evidence for Simulation Theory and the Developmental Role of Language. Child Development, 77, 6, 1842-1853.

Fletcher-Watson, S., Leekam, S.R., Benson, V., Frank, M.C. \& Findlay, J.M. (2009). Eyemovements reveal attention to social information in autism spectrum disorder. Neuropsychologia, 47, 248-257.

Franchak, J.M., Kretch, K.S., Soska, K.C. \& Adolph, K.E. (2011). Head-mounted eyetracking: A new method to describe the visual ecology of infants. Child Development, 82, $1738-1750$.

Frazier Norbury, C., Brock, J., Cragg, L., Einav, S., Griffiths, H., \& Nation, K. (2009). Eyemovement patterns are associated with communicative competence in autistic spectrum disorders. The Journal of Child Psychology and Psychiatry, 50, 834-842.

Freeth, M., Chapman, P., Ropar, D. \& Mitchell, P. (2010). Do Gaze Cues in Complex Scenes Capture and Direct the Attention of High Functioning Adolescents with ASD? Evidence from Eye-tracking. Journal of Autism and Developmental Disorders, 40, 534-547.

Frith, C. D., Frith, U. (2006). The neural basis of mentalizing. Neuron, 50, 531-534.

Hanley, M., McPhillips, M., Mulhern. G. \& Riby, D.M. (2012). Spontaneous attention to faces in Asperger Syndrome using ecologically valid static stimuli. Autism, 17, 754761

Harris, P., Johnson, C.N., Hutton, D., Andrews, G. \& Cooke, T. (1989). Young children’s theory of mind and emotion. Cognition and Emotion, 3, 379-400. 
Hosozawa, M, Tanaka, K., Shimizu, T., Nakano, T. \& Kitazawa, S. (2012). How Children With Specific Language Impairment View Social Situations: An Eye Tracking Study. Paediatrics, 129, 1453-1460.

Howlin, P., Goode, S., Hutton, J. \& Rutter, M. (2004). Adult outcome for children with Autism. Journal of Child Psychology and Psychiatry, 45, 212-229.

Hughes, C., Adlam, A., Happé, F., Jackson, J., Taylor, A., \& Caspi, A. (2000). Good testretest reliability for standard and advanced false-belief tasks across a wide range of abilities. Journal of Child Psychology \& Psychiatry, 41, 483-490.

Jones, W., Carr, K. \& Klin, A. (2008). Absence of preferential looking to the eyes of approaching adults predicts level of social disability in 2-year-old toddlers with autism spectrum disorder. Archives of General Psychiatry, 65, 946-954.

Kelly, D., Walker, R. \& Frazier Norbury, C. (2013). Deficits in volitional oculomotor control align with language status in autism spectrum disorders. Developmental Science, 16, 56-66.

Kleinke, C. L. (1986). Gaze and eye contact: A research review. Psychological Bulletin, 100, 78-100.

Klin, A., Jones, W., Schultz, R., Volkmar, F., \& Cohen, D. (2002a). Defining and quantifying the social phenotype in autism. American Journal of Psychiatry, 159, 895-908.

Klin, A., Jones, W., Schultz, R., Volkmar, F., \& Cohen, D. (2002b). Visual fixation patterns during viewing of naturalistic social situations as predictors of social competence in individuals with autism. Archives of General Psychiatry, 59(9), 809-816.

Klin, A. (2008). In the eye of the beholden: Tracking developmental psychopathology. Journal of the American Academy of Child and Adolescent Psychiatry, 47, 362-363. 
Klin, A. \& Jones, W. (2008). Altered face scanning and impaired recognition of biological motion in a 15-month-old with autism. Developmental Science, 11, 40-46.

Kuhn, G., Kourkoulou, A., \& Leekam, S.R. (2010). How magic changes our expectations about autism. Psychological Science, 21, 1487-1493.

Mundy, P., Delgado, C., Block, L., Venezia, M., Hogan, A. \& Seibert, J. (2003). A manual for the abridged early social communication scales (ESCS). Available through the University of Miami Pychology Department, Coral Gables, Florida.

Mundy, P., Sigman, M., Ungerer, J. \& Sherman, T. (1986). Defining the social deficits of autism: The contribution of non-verbal communication measures. Journal of Child Psychology and Psychiatry, 27, 657-669.

Nadig, A., Lee, I., Singh, L., Bosshart, K. \& Ozonoff, S. (2010). How does the topic of conversation affect verbal exchange and eye gaze? A comparison between typical development and high-functioning autism. Neuropsychologia, 48, 2730-2739.

Nakano, T., Tanak, K., Endo, Y., Yamane, Y., Yamamoto., T., Nakano, Y.,...Kitazawa, S. (2010). Atypical gaze patterns in children and adults with autism spectrum disorders dissociated from developmental changes in gaze behaviour. Proceedings of the Royal Society B-Biological Science, 277, 2935-2943.

Paynter , J. \& Peterson, C. (2010). Language and ToM development in autism versus Asperger syndrome: Contrasting influences of syntactic versus lexical/semantic maturity. Research in Autism Spectrum Disorders, 4, 377-385.

Pelphrey, K.A., Sasson, N.J., Reznick, J.S., Paul, G., Goldman, B.D. \& Piven, J. (2002). Visual scanning of faces in autism. Journal of Autism and Developmental Disorders, 32, 249-261. 
Perner, J. \& Wimmer, H. (1985). John thinks that Mary thinks that - Attribution of $2^{\text {nd }}$ order beliefs by 5-year-old to 10-year-old children. Journal of Experimental Child Psychology, 39, 437-471.

Pruett, J.R., LaMacchia, A., Hoertel, S., Squire, E., Mcvey, K., Todd, R.D., ...Peterson, S.E. (2011). Social and Non-Social Cueing of Visuospatial Attention in Autism and Typical Development. Journal of Autism and Developmental Disorders, 41, 715-731.

Riby, D.M., \& Hancock, P.J.B. (2009a). Looking at movies and cartoons: eye tacking evidence from Williams syndrome and autism. Journal of Intellectual Disability Research, 53, 169-181

Riby, D.M. \& Hancock, P.J.B. (2009b). Do Faces Capture the Attention of Individuals with Williams Syndrome or Autism? Evidence from Tracking Eye Movements. Journal of Autism and Developmental Disorders, 39, 421-431.

Riby, D.M., Doherty-Sneddon, G. \& Whittle, L. (2012). Face-to-face interference in typical and atypical development. Developmental Science, 15, 281-291.

Riby, D.M., Whittle, L. \& Doherty-Sneddon, G. (2012). Physiological reactivity to faces via live and video-mediated communication in typical and atypical development. Journal of Clinical and Experimental Neuropsychology, 34, 385-395.

Rice, K., Moriuchi,J.M., Jones, W. \& Klin, A. (2012). Parsing Heterogeneity in Autism Spectrum Disorders: Visual Scanning of Dynamic Social Scenes in SchoolAged Children. Journal of the American Academy of Child and Adolescent Psychiatry, 51, 238-248.

Rutherford, M. D. \& Towns, A.M. (2008). Scan path differences and similarities during emotion perception in those with and without autism spectrum disorders. Journal of Autism and Developmental Disorder, 38, 1371-1381. 
Semel E, Wiig EH and Secord WA (2006) Clinical Evaluation of Language Fundamentals (4th edition). London: The Psychological Corporation

Senju, A. \& Johnson, M.H. (2009). Atypical eye contact in autism: Models, mechanisms and development. Neuroscience and Biobehavioral Reviews, 33, 1204-1214.

Senju, A., Southgate, V., White, S. \& Frith, U. (2009). Mindblind Eyes: An Absence of Spontaneous Theory of Mind in Asperger Syndrome. Science, 325, 883-885.

Senju, A., Southgate, V., Miura., Y., Matsui, T., Hasegawa,T., Tojo, Y., ...Csibra, G. (2010). Absence of spontaneous action anticipation by false belief attribution in children with autism spectrum disorder. Development and Psychopathology, 22, 353-360.

Speer, L., Cook, A.E., McMahon, W.M. \& Clark, E. (2007). Face processing in children with autism - Effects of stimulus contents and type. Autism, 11, 265-277.

Van der Geest, J. N., Kemner, C., Verbaten, M. N., \& van Engeland, H. (2002). Gaze behavior of children with pervasive developmental disorder toward human faces: A fixation time study. Journal of Child Psychology and Psychiatry and Allied Disciplines, 43(5), 669-678.

Varghese, L.A., Ozmeral, E.J., Best, V. \& Shinn-Cunningham, B.G. (2012). How Visual Cues for when to Listen Aid Selective Auditory Attention. Journal of the Association for Research in Otolaryngology, 13, 359-368.

Volkmar, F., Carter, A., Grossman, J., \& Klin, A. (1997b) Social Development in Autism. In D. Cohen \& F. Volkmar (Eds), Handbook of Autism and Pervasive Developmental Disorders (2 ${ }^{\text {nd }}$ ed.)(pp 173-195). New York: Wiley.

Von Hoftsen, C. \& Gredebäack, G. (2009). The Role of Looking in Social Cognition: Perspectives from Development and Autism. In T. Striano \& V. Reid (Eds), Social Cognition. Development, Neuroscience, and Autism (1 $1^{\text {st }}$ ed.)(pp 237-253). West Sussex: Wiley-Blackwell. 
Wechsler, D., \& Naglieri, J. A. (2006). Wechsler Nonverbal Scale of Ability. San Antonio: Harcourt Assessment

Wimmer, H., \& Hartl, M. (1991). Against the Cartesian view on mind: Young children's difficulty with own false beliefs. British Journal of Developmental Psychology, 9, 125-138.

Wimmer, H., \& Perner, J. (1983). Beliefs about beliefs: Representation and constraining function of wrong beliefs in young children's understanding of deception. Cognition, 13, 103-128.

Yarbus, A. L. (1967). Eye movements and vision. New York: Plenum Press. 
Table 1: Participant Characteristics

\begin{tabular}{llll}
\hline & ASD (N=17) & SLI (N=14) & TD (N=16) \\
& Mean (SD) & Mean (SD) & Mean (SD) \\
\hline Chronological Age $^{\mathbf{a}}$ & $121(25)$ & $115.2(10)$ & $119.6(8)$ \\
Gender (M/F) & $11 / 6$ & $12 / 2$ & $6 / 10$ \\
WNV $^{\mathbf{b}}$ & $92.9(13.764)$ & $93.79(6.852)$ & $95.56(7.174)$ \\
BPVS (raw) $^{\mathbf{c}}$ & $84.2(18)$ & $78(11)$ & $90.6(16)$ \\
BPVS (standard) & $84.5(12.1)$ & $84.4(5.8)$ & $94.06(8.621)$ \\
\hline
\end{tabular}

ASD, Autism Spectrum Disorder; SLI, Specific Language Impairment; TD, Typically Developing.

${ }^{\mathrm{a}}$ Expressed as months.

bAs assessed by the Wechsler Non-Verbal Scale of Ability (WNV; Wechsler \& Naglieri, 2006).

cAs assessed by the British Picture Vocabulary Scale II (BPVS II; Dunn et al.,1997). 
Table 2: First-order correlations between performance on the ToM battery (composite score) and other measures for all participants

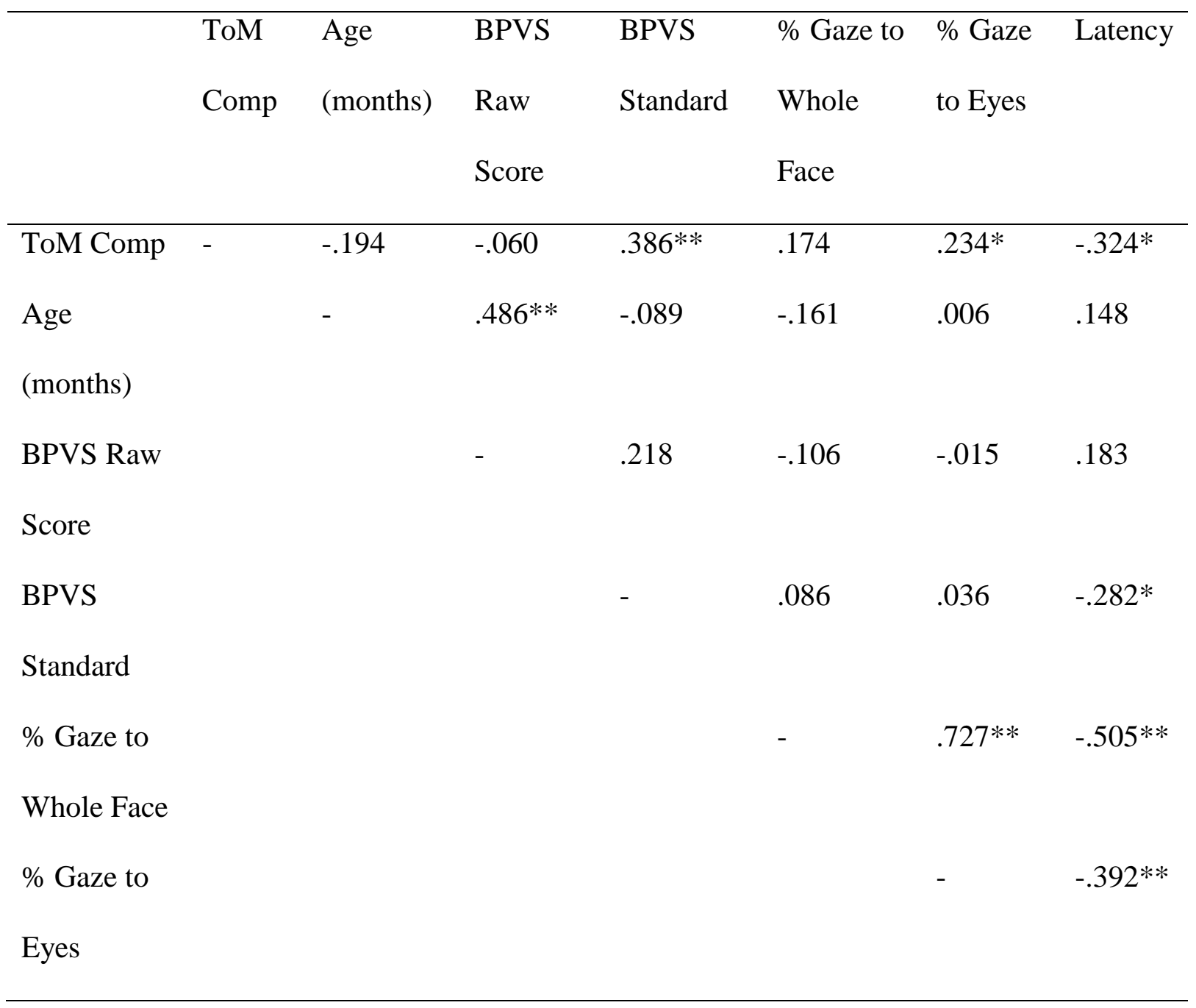

$* \mathrm{p} \leq .05$, all significance values one-tailed.

** $\mathrm{p} \leq .01$, all significance values one-tailed 
Figure 1: Percentage gaze to AOIs for each section and for each group.

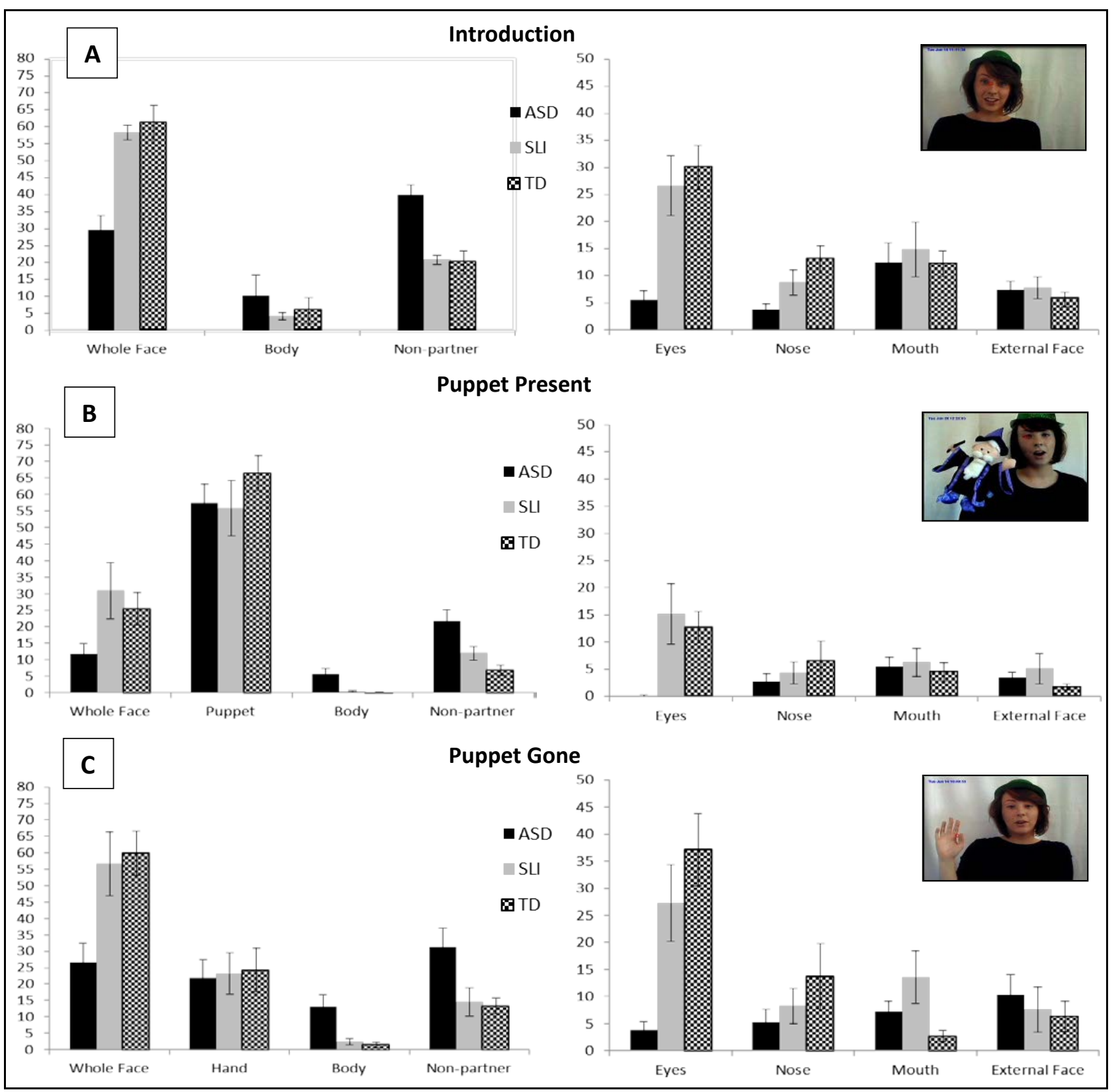


Figure 2: Mean latencies to fixate magician's face for each group following unexpected event

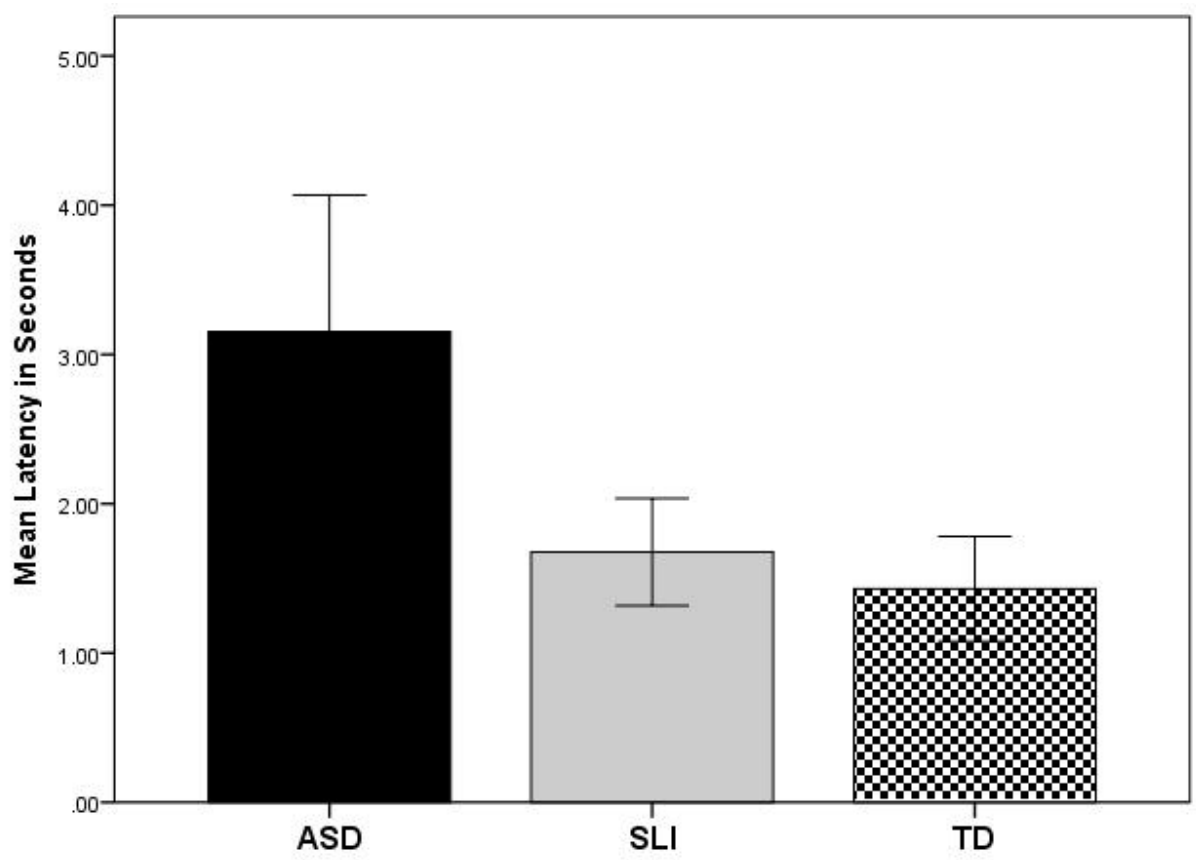

Error bars: $+1-2 \mathrm{SE}$ 
Figure 3: Mean ToM composite scores for each group

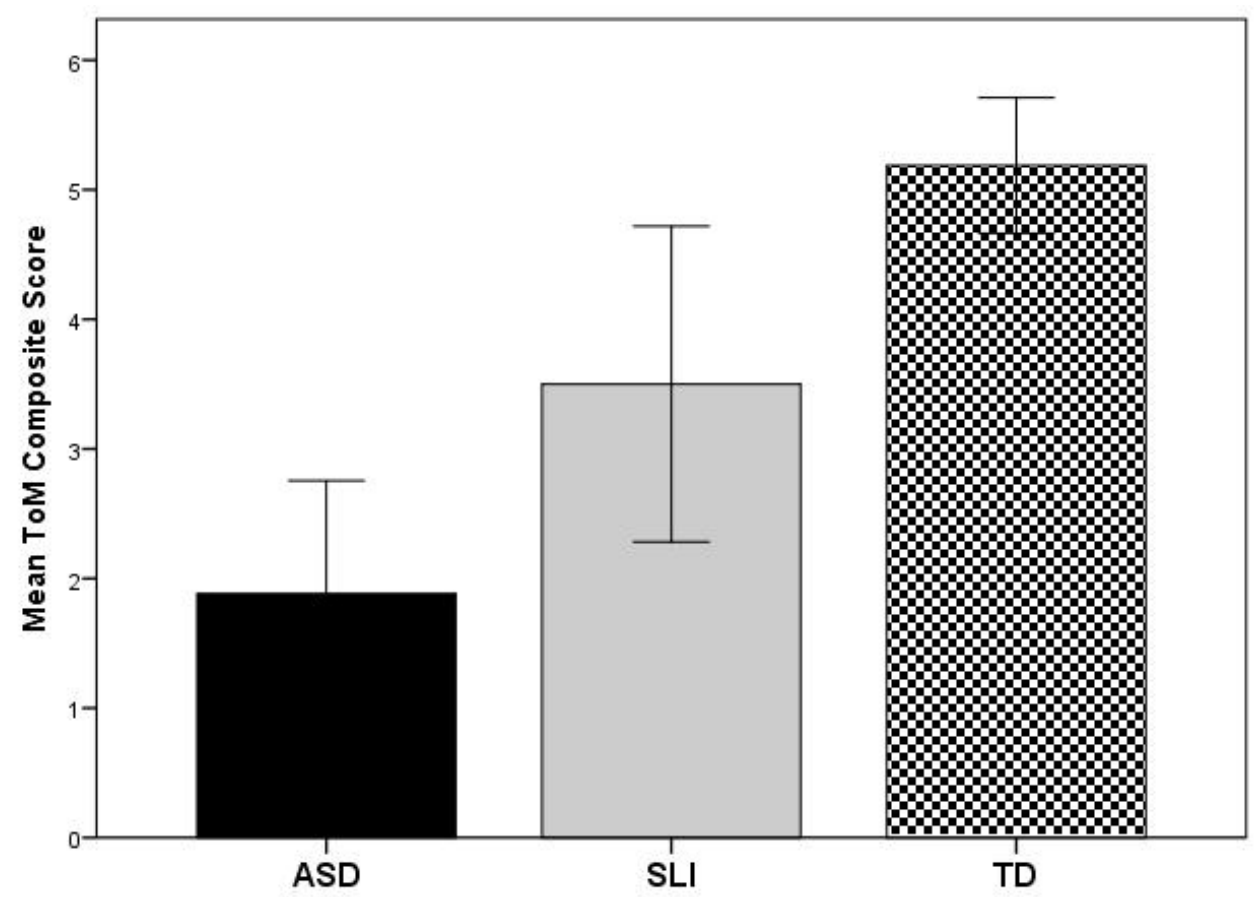

Error bars: +/- $2 \mathrm{SE}$ 